\title{
Comparing multiplex sIgE diagnostics by ALEX and ISAC in pediatric allergy: what are the benefits and drawbacks?
}

Laura Sonneveld $^{1}$, Joyce Emons ${ }^{1}$, Nicolette Arends ${ }^{1}$, Lonneke Landzaat ${ }^{1}$, Sharon Veenbergen $^{1}$, and Marco Schreurs ${ }^{1}$

${ }^{1}$ Erasmus Medical Center

December 7, 2021

\section{Hosted file}

Main text file_Sonneveld_Letter to the editor_ALEXvsISAC.pdf available at https://authorea. com/users/450038/articles/548467-comparing-multiplex-sige-diagnostics-by-alex-and-isacin-pediatric-allergy-what-are-the-benefits-and-drawbacks 
Table 1

\begin{tabular}{|c|c|c|c|c|}
\hline Patient & $\begin{array}{l}\text { Sex } \\
\text { (M/F) }\end{array}$ & $\begin{array}{l}\text { Age } \\
\text { (yr) }\end{array}$ & $\begin{array}{l}\text { Food allergies } \\
\text { (clinical relevant) }\end{array}$ & $\begin{array}{l}\text { Inhalation allergies } \\
\text { (clinical relevant) }\end{array}$ \\
\hline 1 & $M$ & 16 & $\begin{array}{l}\text { Egg, peanut, nuts, coconut, } \\
\text { legumes, wheat, seeds, carrot, } \\
\text { banana, kiwi. }\end{array}$ & $\begin{array}{l}\text { Mites, trees, multiple } \\
\text { animals }\end{array}$ \\
\hline 2 & $\mathrm{M}$ & 12 & Nuts & Trees, grass, multiple animals \\
\hline 3 & M & 12 & $\begin{array}{l}\text { Cow's milk, nuts, certain legumes } \\
\text { (except brown and green beans) }\end{array}$ & Mites, trees, grass, dog \\
\hline 4 & $M$ & 15 & $\begin{array}{l}\text { Nuts, sesame, bean sprouts, } \\
\text { legumes (except brown and green } \\
\text { beans), kiwi }\end{array}$ & Mites \\
\hline 5 & $M$ & 7 & Cow's milk, nuts, kiwi, fish & No clinical relevant \\
\hline 6 & $M$ & 13 & Peanut, nuts (except almond) & $\begin{array}{l}\text { Mites, trees, grass, multiple } \\
\text { animals }\end{array}$ \\
\hline 7 & $\mathrm{~F}$ & 13 & Cow's milk, egg, soya, hazelnut & $\begin{array}{l}\text { Mites, trees, grass, multiple } \\
\text { animals }\end{array}$ \\
\hline 8 & $M$ & 11 & $\begin{array}{l}\text { Peanut, peas, white beans, bean } \\
\text { sprouts, walnut and pecan nut, fish }\end{array}$ & $\begin{array}{l}\text { Mites, trees, grass, multiple } \\
\text { animals }\end{array}$ \\
\hline 9 & $\mathrm{~F}$ & 11 & Cashew nut, pistachio nut, kiwi & Mites, trees, grass, cat \\
\hline 10 & $M$ & 8 & $\begin{array}{l}\text { Peanut, nuts (except almond), } \\
\text { sesame, kiwi }\end{array}$ & $\begin{array}{l}\text { Mites, trees, grass, multiple } \\
\text { animals }\end{array}$ \\
\hline
\end{tabular}


Figure 1

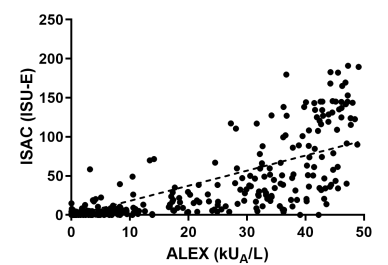

Figure 2
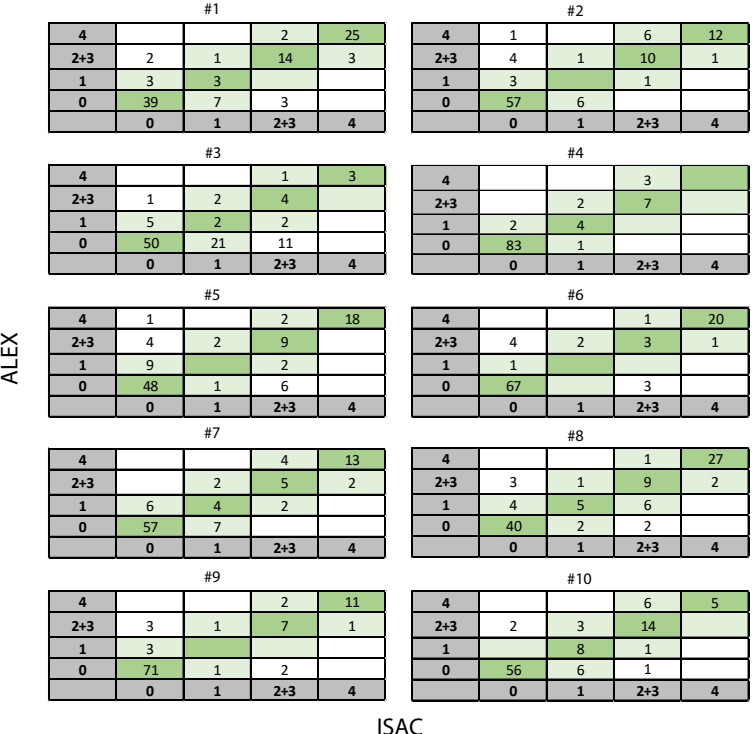